\title{
The Therapeutic Flat as a Means of Orientation and Psychological Adaptation for the Paraplegic's Environment.*
}

\author{
A. Heilporn, M.D., J. Sprimont, M.D., H. Wauthia, M.D., M. Dernies, \\ M.D. \\ Center for Traumatology and Rehabilitation, Place A. Van Gehuchten, 4, Brussels, \\ Belgium
}

After seven years of utilisation of the therapeutic flat annexed to our Occupational Therapy Unit, we are able to consider the therapeutic value.

The flat was described by Bay and Sprimont (1982). It is used for intensive training in all activities of daily living. There is close collaboration with the family and all persons likely to be available to the patient.

Before the patient moves into the flat all necessary information is given concerning nursing care, practical use of technical aids, orthesis, hoists, electric wheelchairs and other equipment. After this the patient may live in the flat alone or with a helper. Each year about 20 patients stay in the flat.

Each situation is different and the aims may be varied but the essential point is that the patient either alone or with his family lives an everyday life situation and becomes fully aware of his possibilities and also his limits.

Alone, confronted with different problems, he must himself or with help put into practice what he has previously learned and find suitable solutions for unforeseen problems. This often helps him to understand the necessity to collaborate with all the different treatments offered to him in order to obtain a maximum of independence. For the younger paraplegic it can prove to him that he may live alone, and thus help parents to accept that their disabled youngster is capable of doing so. Others, on the contrary, may realise that help is necessary, occasionally or permanently.

The helper (usually husband/wife) in the case of a tetraplegic patient is often anxious and afraid of lacking competence and no having the physical capacities or moral resistance necessary. The flat is a relief for them. The semi-secure atmosphere lessens tension and enables them to face positively the different problems involved in caring day and night.

The flat encourages patients to prepare for their future. At an early stage different adaptations concerning the home may be carefully considered and solved, and their return home is then made so much easier.

* Summary of Paper read at the Annual Scientific Meeting of the International Medical Society of Paraplegia, Toledo, Spain, April 1985. 
A second stay at the flat is possible when unforeseen problems arise, for solving technical difficulties or for evaluating permanent or part-time help from brothers, sisters, school friends, and safe guarding friendly relationships.

The personal and family problems that arise following a spinal injury are often helped. An early approach to such situations is essential for a positive outcome. Preliminary information concerning sexual function is given.

Statistics seem to show that authority and trust are the main points of concern. In all cases, separation time must be limited, in spite of hospitalisation. We have found the flat to be an ideal way of revealing to the patient and also to the rehabilitation team the aptitudes necessary for a life as independent as possible, enabling the patient with or without help to progressively adjust himself to his new responsibilities, thus minimising anxiety, misunderstanding and tension, helping difficult relationships, and generally encouraging a well balanced physical and psycholgical evolution.

\section{Reference}

BAy B, SPRimont J 1982 L'appartement Thérapeutique in Troisième Congrès Européen Des Para-Médicaux sur la Paraplégie. Edition Médecine et Hygiène. pp. 189-190. 